\title{
Navigating Dynamic Environments with Trajectory Deformation
}

\author{
Thierry Fraichard and Vivien Delsart \\ INRIA, LIG-CNRS and Grenoble University, France
}

\begin{abstract}
Path deformation is a technique that was introduced to generate robot motion wherein a nominal path, that had been computed beforehand, was continuously deformed on-line in response to unforeseen obstacles. In an effort to improve path deformation, this paper presents a trajectory deformation scheme. The main idea is that by incorporating the time dimension and hence information on the obstacles' future behaviour, quite a number of situations where path deformation would fail can be handled. The trajectory represented as a discrete space-time curve is subject to deformation forces both external (to avoid collision with the obstacles) and internal (to maintain trajectory feasibility and connectivity). The trajectory deformation scheme has been tested successfully on a planar robot with double integrator dynamics moving in dynamic environments.
\end{abstract}

Keywords: mobile robots, autonomous navigation, collision avoidance, motion deformation

\section{Introduction}

Where to move next? is a key question for an autonomous robotic system. This fundamental issue has been largely addressed in the past forty years. Many motion determination strategies have been proposed (see Lavalle (2006) for a review). They can broadly be classified into deliberative versus reactive strategies: deliberative strategies aim at computing a complete motion all the way to the goal, whereas reactive strategies determine the motion to execute during the next few time-steps only. Deliberative strategies have to solve a motion planning problem. They require a model of the environment as complete as possible and their intrinsic complexity is such that it may preclude their application in dynamic environments. Reactive strategies, on the other hand, can operate online using local sensor information: they can be used in any kind of environment whether unknown, changing or dynamic, but convergence towards the goal is difficult to guarantee.

To bridge the gap between deliberative and reactive approaches, a complementary approach has been proposed based upon motion deformation. The principle is simple: a complete motion to the goal is computed first using a priori information. It is then passed on to the robotic system for execution. During the course of the execution, the still-to-be-executed part of the motion is continuously deformed in response to sensor information acquired on-line, thus accounting for the incompleteness and inaccuracies of the a priori world model. Deformation usually results from the application of constraints, both external (imposed by the obstacles) and internal (to maintain motion feasibility and connectivity). Provided that the motion connectivity can be maintained, convergence towards the goal is achieved.

The different motion deformation techniques that have been proposed Quinlan and Khatib (1993); Khatib et al. (1997); Brock and Khatib (2002); Lamiraux et al. (2004); Yang and Brock (2006) all perform path deformation. In other words, what is deformed is a geometric curve, i.e. the sequence of positions that the robotic system is to take in order to reach its goal. The problem with path deformation techniques is that, by design, they cannot take into account the time dimension of a dynamic environment. For instance, in a scenario such as the one depicted in Figure 1, it would be more appropriate to leave the path as it is and adjust the velocity of the robotic system along the path so as to avoid collision with the moving obstacle 
(by slowing down or accelerating). To achieve this, it is necessary to depart from the path deformation paradigm and resort to trajectory deformation instead. A trajectory is essentially a geometric path parametrized by time. It tells us where the robotic system should be, but also when and with what velocity. Unlike path deformation wherein spatial deformation only takes place, trajectory deformation features both spatial and temporal deformations meaning that the planned velocity of the robotic system can be altered thus permitting to handle gracefully situations such as the one depicted in Figure 1.

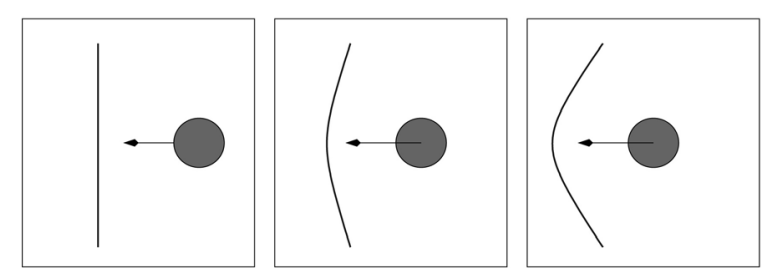

Figure 1. Path deformation problem: in response to the approach of the moving disk, the path is increasingly deformed until it snaps (like an elastic band).

The first trajectory deformation scheme was proposed by one of the authors in Kurniawati and Fraichard (2007). It operates in two stages (collision avoidance and connectivity maintenance stages) and was geared towards manipulator arms. The contribution of this paper is a new trajectory deformation scheme, henceforth called Teddy (for Trajectory Deformer). It operates in one stage only and is designed to handle arbitrary robotic systems.

Teddy is designed to be one component of an otherwise complete autonomous navigation architecture. A motion planning module is required to provide Teddy with the nominal trajectory to be deformed. Teddy operates periodically with a given time period. At each cycle, Teddy outputs a deformed trajectory which is passed to a motion control module that determines the actual commands for the actuators of the robotic system. The paper focuses on Teddy only. It is organised as follows: Teddy is overviewed in Section 2. Its application to the case of a planar robot with double integrator dynamics (subject to velocity and acceleration bounds) is detailed in Section 3. Experimental results are then presented in Section 4.

\section{Overview of the Approach}

\subsection{Notations and Definitions}

Let $\mathcal{A}$ denote a robotic system operating in a workspace $\mathbf{W}\left(\mathbf{R}^{2}\right.$ or $\left.\mathbf{R}^{3}\right)$. $q \in \mathbf{C}$ denote a configuration of $\mathcal{A}$. The dynamics of $\mathcal{A}$ is described by a differential equation of the form:

$$
\dot{s}=f(s, u)
$$

where $s \in \mathbf{S}$ is the state of $\mathcal{A}, \dot{s}$ its time derivative and $u \in \mathbf{U}$ a control. $\mathbf{C}, \mathbf{S}$ and $\mathbf{U}$ respectively denote the configuration space, the state space and the control space of $\mathcal{A}$. Let $\xi:\left[0, t_{f}[\longrightarrow\right.$ $\mathbf{U}$ denote a control input, i.e. a time-sequence of controls. Starting from an initial state $s_{0}$ (at time 0 ) and under the action of a control input $\xi$, the state of $\mathcal{A}$ at time $t$ is denoted by $s\left(s_{0}, \xi, t\right)$. A couple $\left(s_{0}, \xi\right)$ defines a trajectory for $\mathcal{A}$, i.e. a curve in $\mathbf{S} \times \mathbf{T}$ where $\mathbf{T}$ denotes the time dimension.

For the sake of trajectory deformation, a trajectory is discretized in a sequence of nodes. A node is a state-time, it is denoted by $n_{i}=$ $\left(s_{i}, t_{i}\right)$. The discrete trajectory of $\mathcal{A}$ is $\Gamma_{0}=$ $\left\{n_{0}, n_{1} \cdots n_{N}\right\}$ with $n_{0}$ (resp. $\left.n_{N}\right)$ the initial (resp. final) node of the trajectory.

\subsection{Trajectory Deformation Principle}

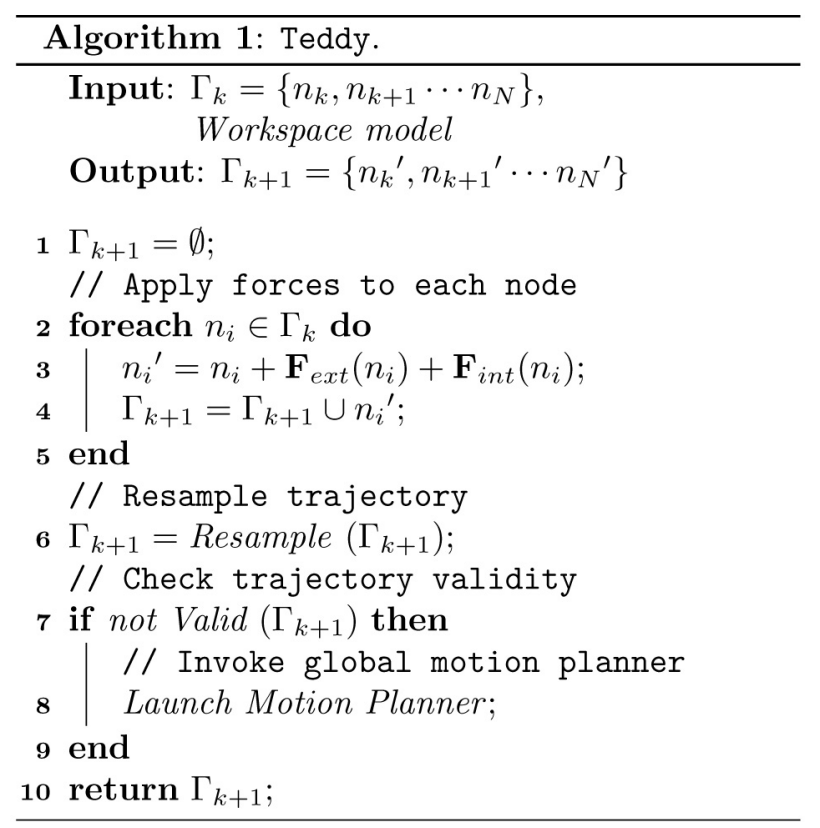

The main steps of Teddy are outlined in Algorithm 1 . Teddy operates periodically with a time 
period of duration $T_{c}$. At time $t_{k}$, it takes as input the still-to-be-executed part of the trajectory $\Gamma_{k}=\left\{n_{k}, n_{k}+1 \cdots n_{N}\right\}$ and an updated model of the workspace. This model includes the position of the obstacles of $\mathbf{W}$ at time $t_{k}$ along with information about their future behaviour. Teddy then deforms $\Gamma_{k}$ in response to the updated position and future behaviour of the obstacles. At time $t_{k+1}=t_{k}+T_{c}$, Teddy outputs a deformed trajectory $\Gamma_{k}+1=\left\{n_{k}^{\prime}, n_{k+1}^{\prime} \cdots n_{N}^{\prime}\right\}$ with $n_{i}^{\prime}$ the updated node corresponding to $n_{i}$.

Like a particle placed in a force field, a node is displaced in response to the application of a force which is the combination of two kind of forces: external and internal. External forces (denoted $\mathbf{F}_{\text {ext }}$ ) are repulsive forces exerted by the obstacles of the environment, their purpose is to deform the trajectory in order to keep it collision-free. They are detailed in subsection 2.3. Internal forces (denoted $\mathbf{F}_{\text {int }}$ ), on the other hand, are aimed at maintaining the feasibility and the connectivity of the trajectory, i.e. to ensure that the deformed trajectory still satisfies the dynamics of $\mathcal{A}$. They are detailed in subsection 2.4.

Now, for the sake of both collision-checking and connectivity evaluation, it is desirable to maintain a regular sampling level along the trajectory. Depending on the situation, nodes are removed or added accordingly. This point is detailed in subsection 2.5.

Finally, it is important to note that, like the path deformation scheme, the trajectory deformation scheme suffers from the following limitation: there is no guarantee that it will produce a collision-free and connected trajectory at each time step; both schemes are heuristic by nature. Failure to produce a valid trajectory typically happens when the topology of $\mathbf{S}$ changes (when a passage is blocked for instance, like when a door is closed). At each time step, the deformed trajectory is therefore checked for collision and connectivity. Should it become invalid, a global motion planner must be invoked to compute a new nominal trajectory. Strictly speaking, the motion planner is not part of Teddy, it is not discussed here.

\subsection{External Forces}

External forces are repulsive forces exerted by the obstacles of the environment for collision avoidance purposes. They are derived from a potential function $\mathbf{V}_{\text {ext }}$. To explicitly take into account the future behaviour of the moving obstacles, $\mathbf{V}_{\text {ext }}$ is defined in the space-time $\mathbf{W} \times \mathbf{T}$ (instead of $\mathbf{S} \times \mathbf{T}$ for efficiency reason). In a manner similar to Brock and Khatib (2002), a set of points $p_{j}$ are selected on the body of $\mathcal{A}$. Each node $n_{i}$ of the trajectory $\Gamma_{k}$ yield a set of control points $c_{i}^{j}=\left(p_{j}, t_{i}\right)$ in $\mathbf{W} \times \mathbf{T}$. For a control point $c^{j}$ corresponding to the configuration $q$ and the state $s$ along the trajectory, $\mathbf{V}_{e x t}$ is defined as:

$\mathbf{V}_{\text {ext }}\left(c^{j}\right)= \begin{cases}k_{\text {ext }}\left(d_{0}-d_{w t}\left(c^{j}\right)\right)^{2} & \text { if } d_{w t}\left(c^{j}\right)<d_{0} \\ 0 & \text { otherwise }\end{cases}$

where $d_{w t}\left(c^{j}\right)$ is the distance from $c^{j}$ to the closest obstacle in $\mathbf{W} \times \mathbf{T} . d_{0}$ is the region of influence around the obstacles and $k_{\text {ext }}$ is a repulsion gain. $d_{w t}$ is a distance function in $\mathbf{W} \times \mathbf{T}$. It is derived from the Euclidean distance by scaling the space versus the time dimension. In $\mathbf{R}^{2}$ for instance, the distance $d_{w t}$ between $\left(x_{0}, y_{0}, t_{0}\right)$ and $\left(x_{1}, y_{1}, t_{1}\right)$ is given by:

$$
\begin{aligned}
d_{w t}^{2}= & w_{s}^{2}\left(x_{1}-x_{0}\right)^{2}+w_{s}^{2}\left(y_{1}-y_{0}\right)^{2} \\
& +w_{t}^{2}\left(t_{1}-t_{0}\right)^{2}
\end{aligned}
$$

with $w_{s}$ (resp. $w_{t}$ ) the spatial (resp. temporal) weight. The force resulting from this potential function acting on $c^{j}$ is then defined as:

$$
\begin{aligned}
\mathbf{F}_{\text {ext }}^{w t}\left(c_{j}\right) & =-\nabla \mathbf{V}_{\text {ext }}\left(c_{j}\right) \\
& =k_{\text {ext }}\left(d_{0}-d_{w t}\left(c_{j}\right)\right) \frac{\mathbf{d}}{\|\mathbf{d}\|}
\end{aligned}
$$

where $\mathbf{d}$ is the vector between $c$ and the closest obstacle point. Now, $\mathbf{F}_{e x t}^{w t}$ has to be mapped into $\mathbf{S} \times \mathbf{T}$. The forces defined in $\mathbf{W} \times \mathbf{T}$ by each control point $c_{j}$ yield a force in $\mathbf{C} \times \mathbf{T}$ defined as follows:

$$
\mathbf{F}_{\text {ext }}^{c t}(q, t)=\sum_{j=1}^{r} J_{c^{j}}^{T}(q, t) \mathbf{F}_{\text {ext }}^{w t}\left(c^{j}\right)
$$

where $J_{c^{j}}^{T}(q, t)$ represents the Jacobian at point $c^{j}$ : 


$$
J_{c^{j}}^{T}(q, t)=\left(\begin{array}{cccc}
\frac{\partial q^{1}}{\partial p_{j}^{1}} & \cdots & \frac{\partial q^{1}}{\partial p_{m}^{j}} & 0 \\
\vdots & \ddots & \vdots & \vdots \\
\frac{\partial q^{n}}{\partial p_{i}^{j}} & \cdots & \frac{\partial q^{n}}{\partial p_{m}^{j}} & 0 \\
0 & \cdots & 0 & 1
\end{array}\right)
$$

with $m$ the dimension of $\mathbf{W}, p_{l}^{j}$ the $l^{\text {th }}$ coordinate of $p^{j}, n$ the dimension of $\mathbf{C}$ and $q^{l}$ the $l^{\text {th }}$ coordinate of $q$. The final mapping into $\mathbf{S} \times \mathbf{T}$ that yields $\mathbf{F}_{\text {ext }}(n)=\mathbf{F}_{\text {ext }}(s, t)$ is carried out by leaving the remaining parameters of $s$ unchanged.

\subsection{Internal Forces}

The external forces defined above push each node of the trajectory away from the obstacles if they are inside their influence region. Internal forces are introduced to ensure that the trajectory remains connected, i.e. that there exists a trajectory verifying the dynamics of $\mathcal{A}$ between two consecutive nodes of the trajectory. Trajectory connectivity is related to the concepts of forward and backward reachability. The set of states that are reachable from a given state $s_{0}$ are defined as (forward-reachability):

$$
\mathcal{R}\left(s_{0}\right)=\left\{s_{f} \in \mathbf{S} \mid \exists \xi, \exists t, s\left(s_{0}, \xi, t\right)=s_{f}\right\}
$$

Likewise, the set of states from which it is possible to reach a given state $s_{0}$ are defined as (backward-reachability):

$\mathcal{R}^{-1}\left(s_{0}\right)=\left\{s_{b} \in \mathbf{S} \mid \exists \xi, \exists t, s\left(s_{b}, \xi, t\right)=s_{0}\right\}$

Let $n_{-}, n$ and $n_{+}$denote three consecutive nodes of the trajectory $\Gamma_{k} . \quad \Gamma_{k}$ is connected at $n$ iff $n \in \mathcal{R}\left(n_{-}\right)$and $n_{+} \in \mathcal{R}(n)$. In other words, $n$ must belong to $\mathcal{R}\left(n_{-}\right) \cap \mathcal{R}^{-1}\left(n_{+}\right)$. Now, two cases arise depending on whether the intersection $\mathcal{R}\left(n_{-}\right) \cap \mathcal{R}^{-1}\left(n_{+}\right)$is empty or not (if this intersection is not empty, it means that $n_{-}$and $n_{+}$are connected together also). The next two sections detail how the internal forces are defined in both cases.

\subsubsection{Case 1: $n_{-}$And $n_{+}$Connected}

In that case, the purpose of the internal force is to ensure that $n$ remains within $\mathcal{R}\left(n_{-}\right) \cap \mathcal{R}^{-1}\left(n_{+}\right)$. To that end, a virtual spring is defined between $n$ and a selected point $H$ belonging to
$\mathcal{R}\left(n_{-}\right) \cap \mathcal{R}^{-1}\left(n_{+}\right)$. It yields a potential function $V_{\text {int }}$ defined in the space-time $\mathbf{S} \times \mathbf{T}$ as:

$$
V_{\text {int }}(n)=k_{\text {int }} d_{s t}(n)^{2}
$$

where $d_{s t}(n)$ is the distance between $(n)$ and $H$. It is defined in a manner similar to $d_{w t}$. $k_{\text {int }}$ is an attraction gain.

$$
\mathbf{F}_{\text {int }}(n)=-\nabla V_{\text {int }}(n)=k_{\text {int }} d_{\text {st }}(n) \frac{\mathbf{d}}{\|\mathbf{d}\|}
$$

where $\mathbf{d}$ is the vector between $(n)$ and $H$.

\subsubsection{Case 2: $n_{-}$And $n_{+}$Disconnected}

In that case, $\mathcal{R}\left(n_{-}\right) \cap \mathcal{R}^{-1}\left(n_{+}\right)=\emptyset$ and it is not possible to find a point $H$ belonging to $\mathcal{R}\left(n_{-}\right) \cap \mathcal{R}^{-1}\left(n_{+}\right)$. The solution proposed then is aimed at restoring the connectivity with $n_{-}$ only. To that end, $H$ is simply selected within $\mathcal{R}\left(n_{-}\right)$and $\mathbf{F}_{\text {int }}$ is defined as in subsection 2.4.1. above.

\subsubsection{Selecting $H$}

Depending on whether $n_{-}$and $n_{+}$are connected together (i.e. whether $\mathcal{R}\left(n_{-}\right) \cap \mathcal{R}^{-1}\left(n_{+}\right)$ is empty or not), $H$ should be selected within $\mathcal{R}\left(n_{-}\right) \cap \mathcal{R}^{-1}\left(n_{+}\right)$or $\mathcal{R}\left(n_{-}\right)$. In the former case, a natural choice for $H$ would be the centroid of $\mathcal{R}\left(n_{-}\right) \cap \mathcal{R}^{-1}\left(n_{+}\right)$. In the latter case, $H$ could for instance be defined as the point of $\mathcal{R}\left(n_{-}\right)$which is the closest to $(n)$.

Other choices are possible of course, but the important thing to note is that, in theory, determining $H$ requires, in the worst case, the characterization of the three sets $\mathcal{R}\left(n_{-}\right), \mathcal{R}^{-1}\left(n_{+}\right)$ and $\mathcal{R}\left(n_{-}\right) \cap \mathcal{R}^{-1}\left(n_{+}\right)$. Computing reachable sets for arbitrary robotic systems is a process whose complexity is dependent upon the dimensionality of the system considered and whether its dynamics is linear or not (cf. Asarin et al. (2006), Mitchell (2007)). Since Teddy has a limited time $T_{c}$ only to deform the trajectory, it is therefore critical that Teddy be able to compute $\mathbf{F}_{\text {int }}(n)$ as efficiently as possible. To that end, it is important to exploit as much as possible the properties of the robotic system considered, and in some cases, to resort to various approxima- 
tion or linearization schemes. The case study in Section 3. presents such an approximation scheme.

In the case where $n_{-}$and $n_{+}$are connected, another possibility is to compute a feasible trajectory from $n_{-}$to $n_{+}$and to select, say its intermediate state, to define $H$. Once again, it is the particulars of the robotic systems at hand that determine how the internal forces are actually computed.

\subsection{Trajectory Resampling}

In the course of the deformation process, the nodes of the trajectory may either move away from their neighbours or, on the contrary, move very close to them (whether it be in the spatial or the temporal dimensions). For the sake of both collision-checking and connectivity evaluation, it is desirable to maintain a regular sampling level of the trajectory $\Gamma_{k}$. Depending on the situation, nodes are removed or added accordingly.

Let $n_{-}, n$ and $n_{+}$denote three consecutive nodes of the trajectory $\Gamma_{k}$. A space-time distance similar to $d_{w t}$ is used to compute the distance between two nodes (cf. (2)). To begin with, if the distance between $n_{-}$and $n_{+}$is less than a given threshold, $n$ is removed from $\Gamma_{k}$. Then, the distance between $n_{-}$and $n$ is computed. If is is greater than a given threshold then a new intermediate node $n_{i}$ is added to $\Gamma_{k} . n_{i}$ can be defined as the centroid of $\mathcal{R}\left(n_{-}\right) \cap \mathcal{R}^{-1}(n)$. This node-adding procedure is repeated recursively for both pair of nodes $\left(n_{-}, n_{i}\right)$ and $\left(n_{i}, n\right)$ (in case $n_{-}$and $n$ are really far from one another). The same node-adding procedure is repeated for the nodes $n$ and $n_{+}$.

\section{Case Study: Double Integrator}

To begin with, Teddy has been applied to the case of a $2 \mathrm{D}$ planar robot $\mathcal{A}$ with double integrator dynamics (point mass model). A state of $\mathcal{A}$ is characterized by $(p, v)$ that respectively denote the $2 \mathrm{D}$ position and velocity of $\mathcal{A}: p=(x, y)$ and $v=\left(v^{x}, v^{y}\right)$. The dynamics of $\mathcal{A}$ is given by:

$$
\left(\begin{array}{c}
\dot{p} \\
\dot{v}
\end{array}\right)=\left(\begin{array}{c}
v \\
a
\end{array}\right)
$$

where $a$ denotes the acceleration control applied to $\mathcal{A}$. $|a| \leq a_{\max }$ and $|v| \leq v_{\max }$.

As mentioned earlier in subsection 2.4.3., one key point in the adaptation of Teddy to a particular robotic systems lies in the determination of the point $H$ that is used to compute the internal force $\mathbf{F}_{\text {int }}$. It is important that $H$ can be computed efficiently. Algorithm 2 outlines the way $H$ is computed in our case. The main idea is to compute both $\mathcal{R}\left(n_{-}\right)$and $\mathcal{R}^{-1}\left(n_{+}\right)$for a particular time slice only, namely the intermediate time slice $t=\left(t_{+}-t_{-}\right) / 2$. It is also taking advantage of the fact that it is possible for the system (10) to compute the sets $\mathcal{R}\left(n_{-}\right)$, $\mathcal{R}^{-1}\left(n_{+}\right)$and $\mathcal{R}\left(n_{-}\right) \cap \mathcal{R}^{-1}\left(n_{+}\right)$for each spatial dimension independently.

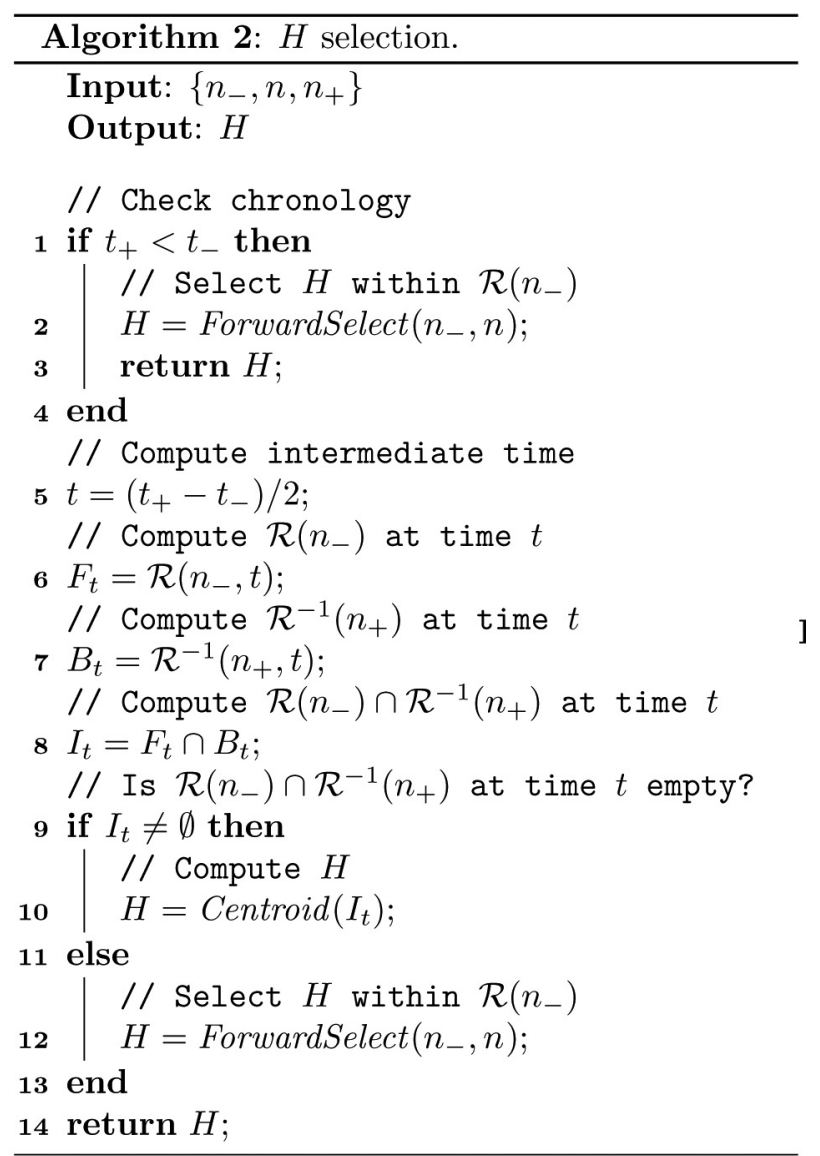

Let us first consider how to compute $\mathcal{R}\left(n_{-}\right)$for the time slice $t$ (henceforth denoted $\mathcal{R}\left(n_{-}, t\right)$ ), and for the $x$-dimension only, i.e. in the case where $n_{-}=\left(x_{-}, v_{-}^{x}, t_{-}\right)$. The $y$-dimension is dealt with similarly and so is the computation of $\mathcal{R}^{-1}\left(n_{+}, t\right)$. 


\section{Computing $\mathcal{R}\left(n_{-}, t\right)$}

First, the extremal positions reachable at time $t$ from $n_{-}$are computed. It is easily achieved by integrating forward (10) while applying the extremal control $\pm a_{\max }$ (until $\pm v_{\max }$ is reached). Let $p_{\min }(t)$ and $p_{\max }(t)$ denote these extremal positions. Then, for a discrete set of positions $p_{i} \in\left[p_{\min }(t) ; p_{\max }(t)\right]$, we compute the corresponding extremal velocities $v_{\min }\left(p_{i}, t\right)$ and $v_{\max }\left(p_{i}, t\right)$. Now, the convex hull of the corresponding set of position-velocity pairs yields a 2D polygonal approximation of $\mathcal{R}\left(n_{-}, t\right) \cdot \mathcal{R}^{-1}$ $\left(n_{+}, t\right)$ is computed in a similar manner.

\section{Computing $\mathcal{R}\left(n_{-}, t\right) \cap \mathcal{R}^{-1}\left(n_{+}, t\right)$}

Both $\mathcal{R}\left(n_{-}, t\right)$ and $\mathcal{R}^{-1}\left(n_{+}, t\right)$ are represented by $2 \mathrm{D}$ polygons of the position-velocity space. A straightforward polygon intersection yields $\mathcal{R}\left(n_{-}, t\right) \cap \mathcal{R}^{-1}\left(n_{+}, t\right)$. If $\mathcal{R}\left(n_{-}, t\right) \cap \mathcal{R}^{-1}\left(n_{+}, t\right)$ is not empty then its centroid is computed, it becomes $H$ (line 10 of Algorithm 2).

\section{Selecting $H$ within $\mathcal{R}\left(n_{-}\right)$}

Now, if $\mathcal{R}\left(n_{-}, t\right) \cap \mathcal{R}^{-1}\left(n_{+}, t\right)$ is empty, $H$ must be selected within $\mathcal{R}\left(n_{-}\right)$in order to try to maintain the connectivity between $n_{-}$and $n$. To that end, a discrete set of time instants $t_{j}>t_{-}$is defined and the corresponding reachable sets $\mathcal{R}\left(n_{-}, t_{j}\right)$ are computed as above. Their centroids $H_{j}$ are computed as well. Finally the point $H_{j}$ whose distance to $n$ is minimal becomes $H$ (lines 2 and 12 of Algorithm 2).

\section{Experimental Results}

Teddy has been implemented in $\mathrm{C}++$ and tested on an Intel Pentium 4 desktop PC ( $3 \mathrm{GHz}, 1 \mathrm{~GB}$ RAM, Linux OS). Teddy has been evaluated in different scenarios. At each time cycle, Teddy is provided with a new model of the environment under the form of a list of a fixed and moving obstacles $\left.\mathcal{B}_{i}\right)$. The moving obstacles are moving randomly but the model of the future assumes they maintain a constant linear velocity. Figure 2 illustrates in a visual manner how Teddy operates.

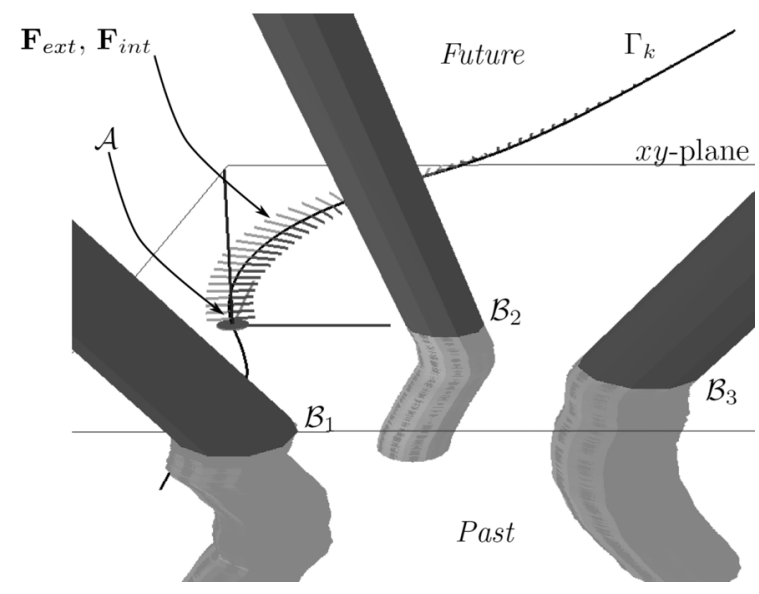

Figure 2. Teddy's principle visualized in a scenario involving three moving disk obstacles $\mathcal{B}_{i}, i=1-3$. The time dimension is pointing upward. The past lies below the $x y$-plane (the present) and the future lies above. The obstacles are moving randomly, but the model of the future assumes that they maintain a constant linear velocity. The internal and external forces acting upon the nodes of the trajectory $\Gamma_{k}$ are represented by vectors.

\section{1. "Cutting" Scenario}

To emphasize the interest of trajectory deformation vs path deformation, a "cutting" scenario similar to the one depicted in Figure 1 has been considered first. This scenario was selected because it is problematic for classical path deformation schemes.

Teddy relies upon a number of parameters to operate properly: the repulsion gain $k_{\text {ext }}$, the attraction gain $k_{\text {int }}$ and the distance functions $d_{w t}$ and $d_{s t}$. The two examples presented below have been selected to illustrate the importance of the distance function $d_{w t}$ on the performance of Teddy. Recall that $d_{w t}$ is used to determine the distance between a trajectory node and the closest obstacle in $\mathbf{W} \times \mathbf{T}$ (cf. subsection 2.3). In both examples, the initial trajectory had a duration of 20 s and the discrete trajectory contained 320 nodes. Teddy would run at $28 \mathrm{~Hz}$.

For the same scenario, two very different deformation patterns can be obtained by properly selecting the weights $w_{s}$ and $w_{t}$ in (2). The first example is obtained by giving more weight to $w_{s}$ thereby allowing more important spatial deformations to take place (Figure 3 ). In this case, $\mathcal{A}$ has time to pass before the obstacle crosses its path. The path component of the trajectory is deformed downwards for safety reasons whereas the velocity component is only slightly modified. 


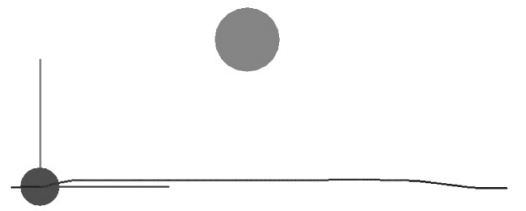

(a) space view, $t=0$

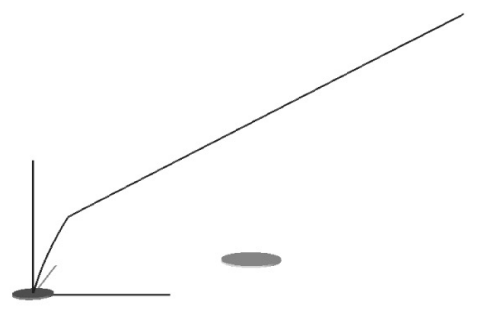

(d) time view, $\mathrm{t}=0$

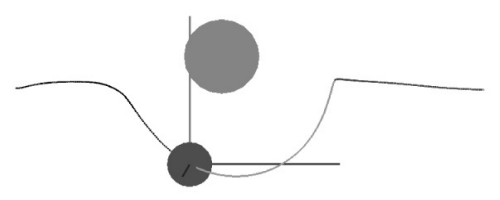

(b) space view, $\mathrm{t}=10$

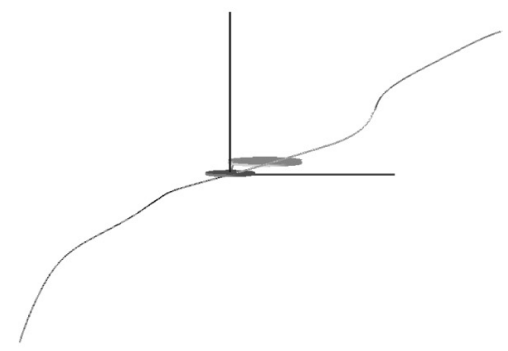

(e) time view, $\mathrm{t}=10$

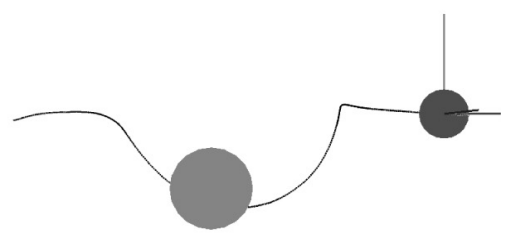

(c) space view, $\mathbf{t}=20$

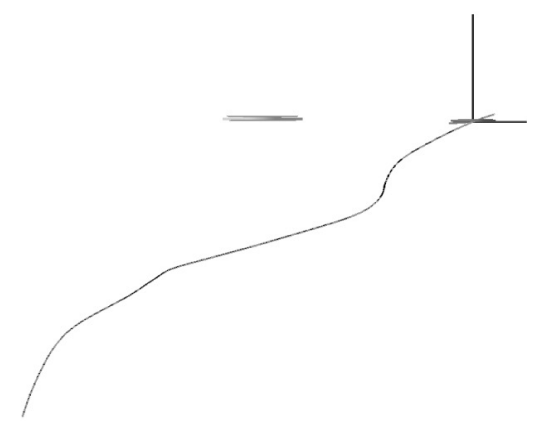

(f) time view, $\mathrm{t}=20$

Figure 3. "Cutting" scenario (spatial deformation): $\mathcal{A}$ is moving from the left to the right, the obstacle is moving downwards. The top snapshots depict the path at different time instant $(x \times y$ view $)$. The bottom snapshots depict the velocity profile at the same instants $(x \times t$ view).

The second example, on the other hand, is obtained by giving more weight to $w_{t}$ thereby allowing more important temporal deformations to take place (Figure 4$)$. In this case, $\mathcal{A}$ lets the obstacle cross its path before proceeding. The path component of the trajectory is only slightly modified whereas the velocity component is largely deformed so as to allow $\mathcal{A}$ to slow down and stop in order to give way to the obstacle.

These two examples have shown the influence of the choice of the parameters in the final performance of Teddy. They have also illustrated the advantage of trajectory deformation versus path deformation.

\subsection{Miscellaneous Scenarios}

Afterwards, Teddy was tested on different scenarios featuring up to 10 obstacles moving randomly (their linear velocity change at each time cycle). Three runs with different settings for the weights $w_{s}$ and $w_{t}$ are illustrated in Figures 5 to 7. In Figure 5, $w_{s}$ is the most important thereby allowing more important spatial deformations to take place. In Figure $6, w_{t}$ is the most important thereby allowing more important temporal deformations to take place. In Figure $7, w_{s}$ and $w_{t}$ are balanced, both spatial and temporal deformations take place. Finally, Teddy was tested on a scenario featuring both fixed and moving obstacles (Figure 8).

\subsection{Performances of Teddy}

From a complexity point of view, it grows linearly with the number of nodes and the number of obstacles. Table 1 gives the running time of one deformation cycle for different numbers of nodes and obstacles. These results show the ability of Teddy to operate efficiently even in highly cluttered environments.

\begin{tabular}{|c|c|c|c|c|c|}
\hline \multirow{2}{*}{$\begin{array}{c}\text { number of } \\
\text { obstacles }\end{array}$} & \multicolumn{5}{|c|}{ number of nodes } \\
\cline { 2 - 6 } & 50 & 100 & 180 & 250 & 320 \\
\hline \hline 1 & 6 & 11 & 20 & 27 & 35 \\
\hline 3 & 44 & 48 & 68 & 70 & 73 \\
\hline 10 & 49 & 88 & 135 & 199 & 229 \\
\hline
\end{tabular}

Table 1. Running time (in ms) of one deformation cycle as a function of the number of nodes and obstacles 


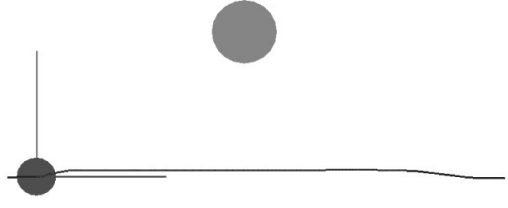

(a) space view, $\mathrm{t}=0$

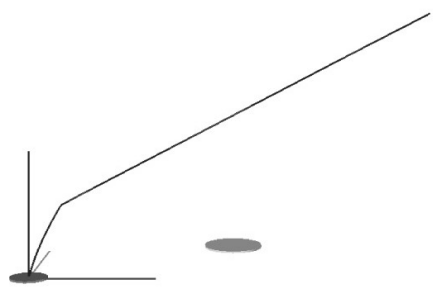

(d) time view, $\mathrm{t}=0$

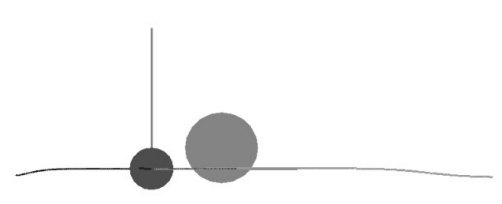

(b) space view, $\mathrm{t}=10$

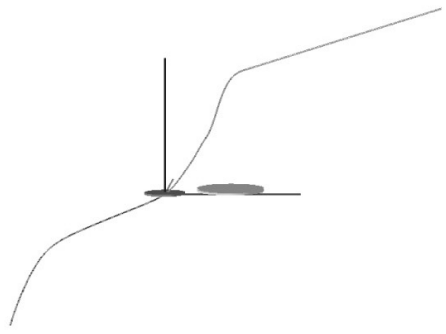

(e) time view, $\mathrm{t}=10$

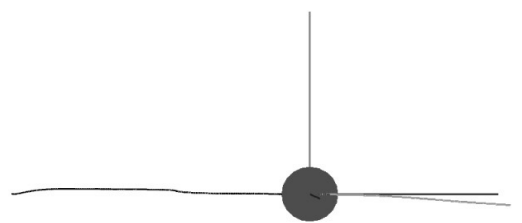

(c) space view, $\mathrm{t}=35$

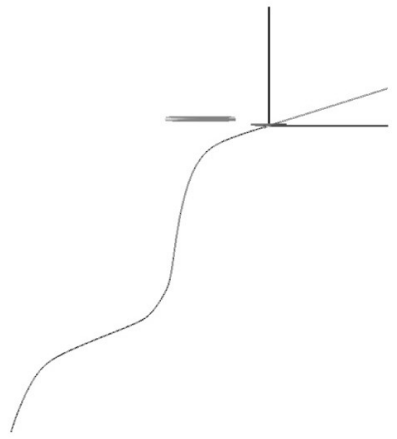

(f) time view, $\mathrm{t}=35$

Figure 4. "Cutting" scenario (temporal deformation): $\mathcal{A}$ is moving from the left to the right, the obstacle is moving downwards. The top snapshots depict the path at different time instant $(x \times y$ view $)$. The bottom snapshots depict the velocity profile at the same instants $(x \times t$ view $)$.

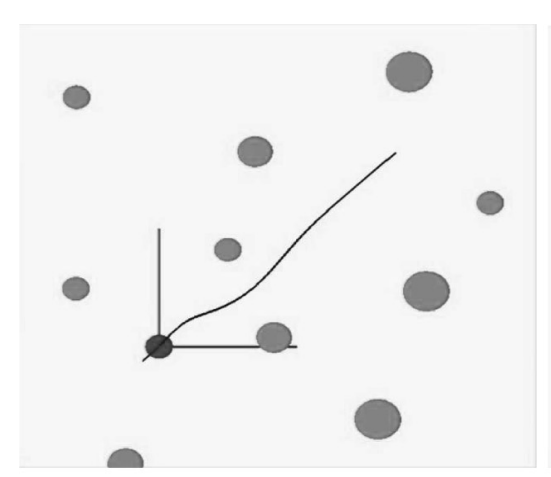

(a) space view, $t=0$
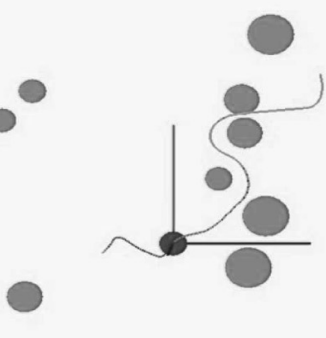

(b) space view, $\mathrm{t}=10$

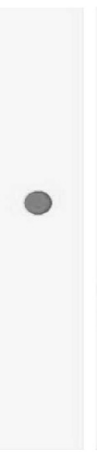

0

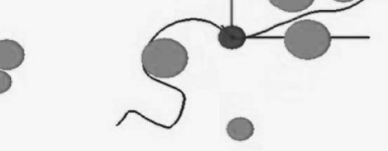

(c) space view, $\mathrm{t}=20$

Figure 5. Multi-disk scenario (spatial deformation): $\mathcal{A}$ is moving from the bottom-left corner to the top-right corner.

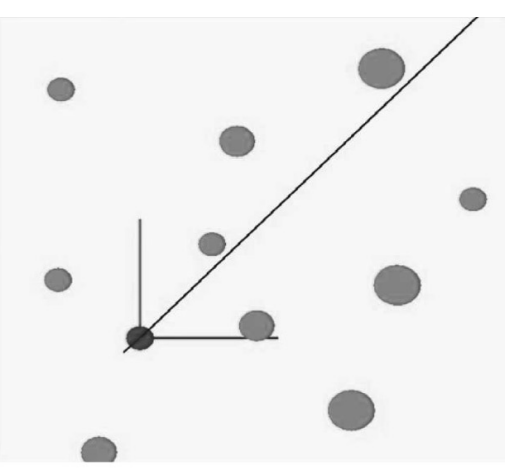

(a) space view, $\mathrm{t}=0$

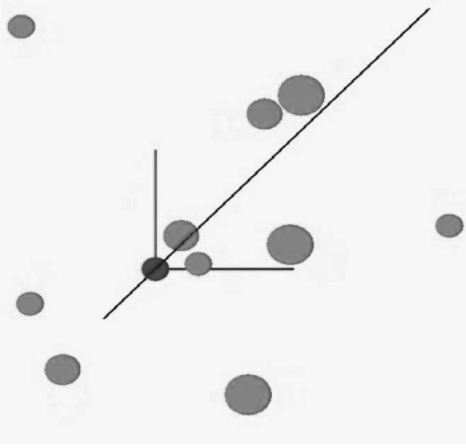

(b) space view, $\mathrm{t}=10$

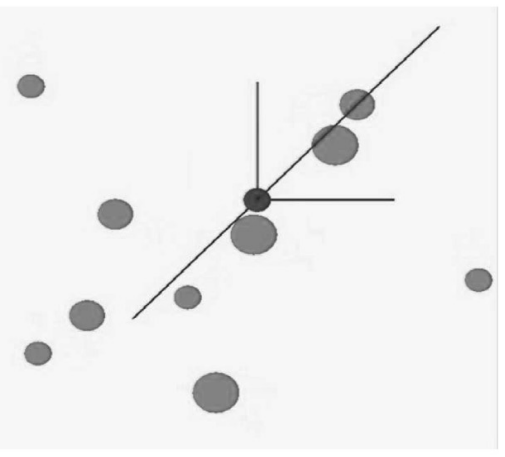

(c) space view, $\mathrm{t}=35$

Figure 6. Multi-disk scenario (temporal deformation):

$\mathcal{A}$ is moving from the bottom-left corner to the top-right corner. 


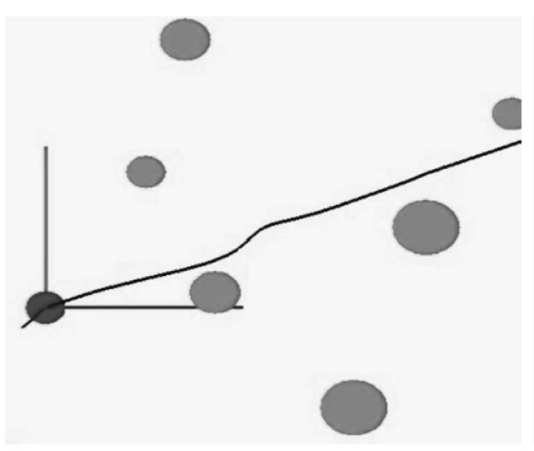

(a) space view, $\mathrm{t}=0$

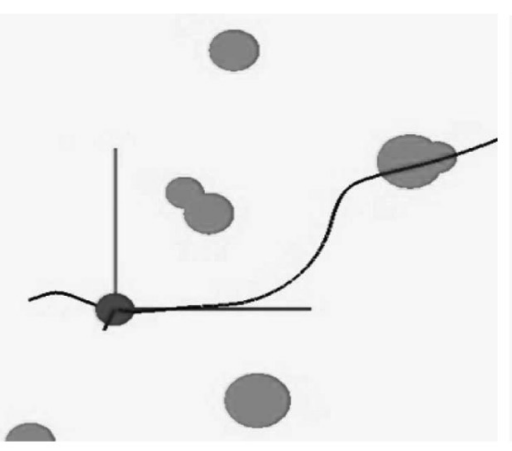

(b) space view, $\mathrm{t}=10$

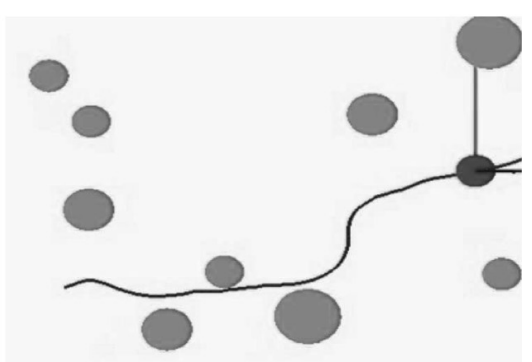

(c) space view, $t=40$

Figure 7. Multi-disk scenario (spatio-temporal deformation): $\mathcal{A}$ is moving from the bottom-left corner to the top-right corner.

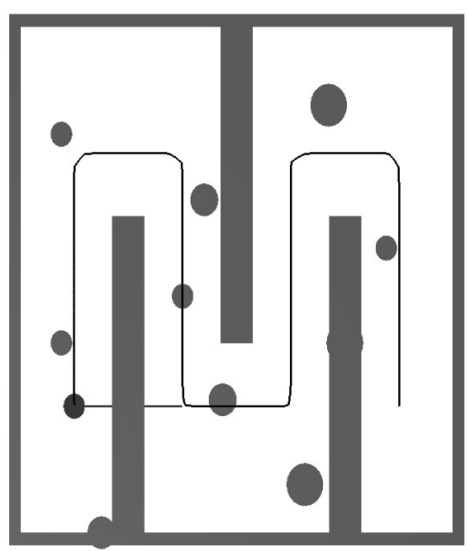

(a) space view, $t=0$

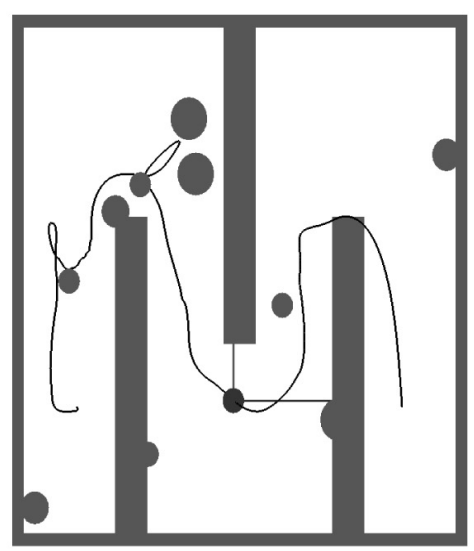

(d) space view, $t=21$

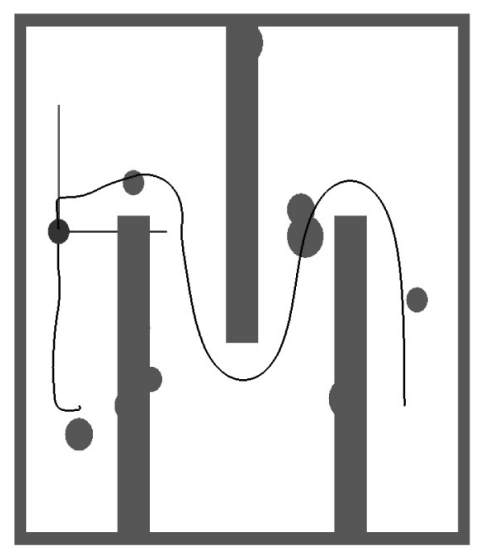

(b) space view, $\mathrm{t}=7$

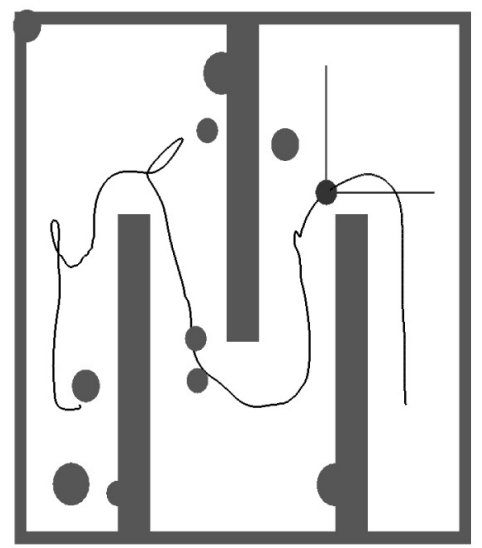

(e) space view, $\mathrm{t}=28$

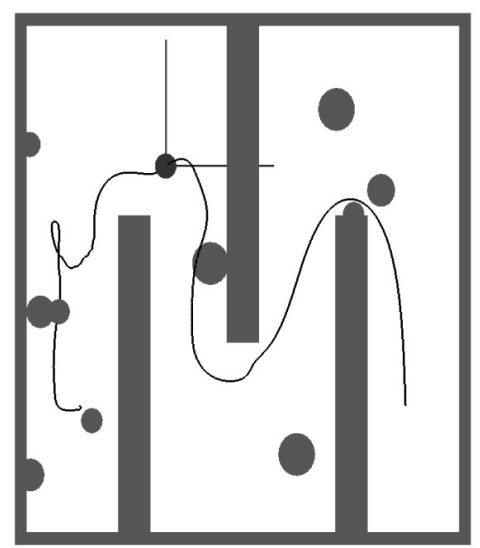

(c) space view, $t=14$

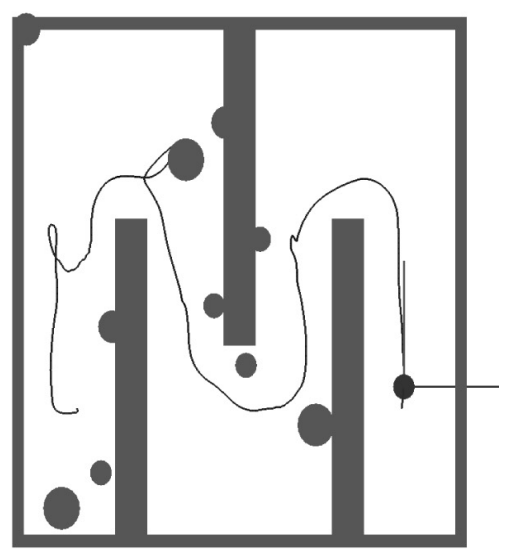

(f) space view, t $=35$

Figure 8. Maze scenario (spatio-temporal deformation): $\mathcal{A}$ is moving from the left to right among fixed polygonal obstacles and moving disks. 


\section{Conclusion and Future Works}

The paper has presented Teddy, a trajectory deformation scheme. Given a nominal trajectory reaching a given goal, Teddy deforms it reactively in response to updated information about the environment's obstacles. Teddy can handle robotic systems with arbitrary dynamics. It has been applied to the case of a 2D double integrator system and tested in various situations. Because, Teddy explicitly takes into account information on the future behaviour of the obstacles, it is able to handle situations that are problematic for classical path deformation schemes. In the future, it is planned to consider other robotic systems, e.g. differential drive/car-like vehicles, and to further optimize Teddy. Considering, for instance, that the knowledge about the future behaviour is less reliable in the distant future, it could be interesting to monotonically decrease the influence of the obstacles with respect to time. Last but not least, Teddy remains to be integrated within a global navigation architecture and tested on an actual robotic system. It is planned to do so on the architecture and the vehicle presented in Chen et al. (2007).

\section{Acknowledgments}

This work was supported by the French Ministry of Defence (DGA Doctoral Grant) and by the European Commission contracts "Cybercars-2 FP6-IST-2004-028062" and "Have-It FP7-IST2007-212154".

\section{References}

[1] Asarin, E., Dang, T., Frehse, G., Girard, A., LE GuERNIC, C. AND MALER, O., Recent progress in continuous and hybrid reachability analysis. In: Proc. of the IEEE Int. Conf. on Computer Aided Control Systems Design, Munich (DE), Oct. 2006.

[2] Brock, Oliver AND KhatiB, Oussama, Elastic Strips: A Framework for Motion Generation in Human Environments. The International Journal of Robotics Research 21 (12), 2002.

[3] Chen, G., Fraichard, Th. And MartinezGOMEZ, L., A Real-Time Autonomous Navigation Architecture. In: Proc. of the IFAC Symp. on Intelligent Autonomous Vehicles, Toulouse (FR), Sep. 2007.
[4] Khatib, M. and JaOuni, H. And Chatila, R. AND LAUMOND, J.P., Dynamic Path Modification for Car-like Nonholonomic Mobile Robots. In: Proceedings of the 1997 IEEE - International Conference on Robotics and Automation, Albuquerque, New Mexico, April, 1997.

[5] Kurniawati, H. AND Fraichard, Th., From Path to Trajectory Deformation. In: Proc. of the IEEERSJ Int. Conf. on Intelligent Robots and Systems, San Diego, CA (US), Oct. 2007.

[6] LAMIRAUX, F. AND BONNAFOUS, D. AND LEFEBVRE, O., Reactive Path Deformation for Nonholonomic Mobile Robots. In: IEEE Trans. on Robotics and Automation, 20 (6), Dec. 2004.

[7] Lavalle, S. M., Planning Algorithms. Cambridge University Press, 2006.

[8] Mitchell, I., Comparing forward and backward reachability as tools for safety analysis. In: Hybrid Systems: Computation and Control, Lecture Notes in Computer Science 4416, Springer, 2007.

[9] Quinlan, S. And KhatiB, O., Elastic Bands: Connecting Path Planning and Control. In Proc. of the IEEE Int. Conf. on Robotic and Automation, Atlanta, GA(USA), May, 1993.

[10] YANG, Y. AND BROCK, B., Elastic Roadmaps: Globally Task-Consistent Motion for Autonomous Mobile Manipulation. In: Proc. of the Int. Conf. Robotics: Science and Systems, Philadelphia PA (US), Aug. 2006.

Received: November, 2007 Revised: May, 2008 Accepted: July, 2008

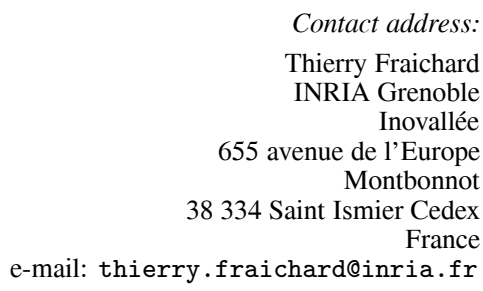

THIERRY FRAICHARD is a Research Scientist in the INGRIA Grenoble Rhône-Alpes Research Center in France. He does research on motion autonomy for vehicles with a special emphasis on safe navigation, motion planning (for nonholonomic systems in dynamic environments and in the presence of uncertainty), prediction of the future motion of moving objects, and the design of control architectures for autonomous vehicles. He received his Ph.D ('92) and his Accreditation to Supervise Research ('06) in Computer Science from the Institut National Polytechnique de Grenoble (INPG), France.

VIVIEN DELSART is currently a Ph.D student at INPG. He received a Master Degree ('07) in Computer Science from INPG for his work on trajectory deformation. 